\title{
EFSUMB 2020 Proposal for a Contrast-Enhanced Ultrasound-Adapted Bosniak Cyst Categorization - Position Statement
}

\section{EFSUMB 2020 - Vorschlag für eine an den kontrastverstärkten Ultraschall adaptierte Bosniak-Klassifikation von Zysten - Eine Stellungnahme}

Authors

Vito Cantisani ${ }^{1}$, Michele Bertolotto ${ }^{2}$, Dirk-André Clevert ${ }^{3}$, Jean-Michel Correas ${ }^{4}$, Francesco Maria Drudi ${ }^{5}$, Thomas Fischer ${ }^{6}$, Odd Helge Gilja ${ }^{7}$, Antonio Granata ${ }^{8}$, Ole Graumann ${ }^{9}$, Christopher J. Harvey ${ }^{10}$, Andre Ignee ${ }^{11}$, Christian Jenssen ${ }^{12}$, Markus Herbert Lerchbaumer ${ }^{13}$, Matthew Ragel ${ }^{14}$, Adrian Saftoiu ${ }^{15}$, Andreas L. Serra ${ }^{16}$, Konrad Friedrich Stock ${ }^{17}$, Jolanta Webb ${ }^{18}$, Paul S. Sidhu ${ }^{19}$

Affiliations

1 Department of Radiology, “Sapienza” University of Rome, Rome, Italy

2 Department of Radiology, University of Trieste, Ospedale di Cattinara, Trieste, IT

3 Department of Clinical Radiology, University of MunichGroßhadern Campus, Munich, Germany

4 Service de Radiologie adultes, Hôpital Necker, Université Paris Descartes, Paris, France

5 Department of Radiology, University La Sapienza, Italy

6 Department of Radiology, University Berlin, Charité, Berlin, Germany

7 Haukeland University Hospital, National Centre for Ultrasound in Gastroenterology, Bergen, Norway

8 Nephrology and Dialysis Unit, Emergency Hospital "Cannizzaro", Catania - Italy

9 Research and Innovation Unit of Radiology, University of Southern Denmark, Odense C, Denmark

10 Department of Imaging, Imperial College NHS Healthcare Trust, London, United Kingdom of Great Britain and Northern Ireland

11 Innere Medizin 2, Caritas-Krankenhaus, Bad Mergentheim, Germany

12 Klinik für Innere Medizin, Krankenhaus Märkisch Oderland Strausberg/Wriezen, Germany

13 Department of Radiology, Charité Centrum 6 Diagnostische und interventionelle Radiologie und Nuklearmedizin, Berlin, Germany

14 Radiology Department, Aintree University Hospitals NHS Foundation Trust, Liverpool, United Kingdom of Great Britain and Northern Ireland

15 Research Center in Gastroenterology and Hepatology, University of Medicine and Pharmacy Craiova, Romania

16 Department of Internal Medicine and Nephrology, Klinik Hirslanden, Zürich, Switzerland

17 Abteilung für Nephrologie, Klinikum rechts der Isar der TU München, München, Germany
18 Radiology Department, Aintree University Hospitals NHS Foundation Trust, Liverpool, United Kingdom of Great Britain and Northern Ireland

19 Department of Radiology, King's College Hospital London, United Kingdom of Great Britain and Northern Ireland

Key words

urinary tract, renal cyst, Bosniak classification, CEUS, cystic renal lesions

received 24.04.2020

accepted 20.10.2020

published online 11.12 .2020

Bibliography

Ultraschall in Med 2021; 42: 154-166

DOI 10.1055/a-1300-1727

ISSN $0172-4614$

(c) 2020. Thieme. All rights reserved.

Georg Thieme Verlag KG, Rüdigerstraße 14,

70469 Stuttgart, Germany

Correspondence

Dr. Vito Cantisani

Department of Radiology, "Sapienza” University of Rome,

Viale Regina Elena, 324, 00161 Rome, Italy

Tel.: +39/3471743947

Fax: $+39 / 064455602$

vito.cantisani@uniroma1.it

\section{ABSTRACT}

The well-established Bosniak renal cyst classification is based on contrast-enhanced computed tomography determining the malignant potential of cystic renal lesions. Ultrasound has not been incorporated into this pathway. However, the development of ultrasound contrast agents coupled with the superior resolution of ultrasound makes it possible to redefine the imaging of cystic renal lesions. In this position statement, an EFSUMB Expert Task Force reviews, analyzes, and describes the accumulated knowledge and limitations and presents the 
current position on the use of ultrasound contrast agents in the evaluation of cystic renal lesions.

\section{ZUSAMMENFASSUNG}

Die gut etablierte Bosniak-Klassifikation von Nierenzysten basiert auf der kontrastverstärkten Computertomografie, die das maligne Potenzial zystischer Nierenläsionen bestimmt. Die Sonografie wurde bei diesem Verfahren nicht berücksich- tigt. Die Entwicklung von Ultraschallkontrastmitteln in Verbindung mit der überlegenen Auflösung im Ultraschall ermöglicht es jedoch, die Bildgebung zystischer Nierenläsionen neu zu definieren. In dieser Stellungnahme überprüft, analysiert und beschreibt eine Expertengruppe der EFSUMB das gesammelte Wissen und die Grenzen und präsentiert die aktuelle Position bezüglich des Einsatzes von Ultraschallkontrastmitteln bei der Bewertung zystischer Nierenläsionen.

\section{Introduction}

It remains a challenge for an imaging modality to accurately differentiate benign from malignant complex renal cysts. This is important as up to $6 \%$ of all asymptomatic renal lesions are cystic renal cell carcinomas [1, 2]. Bosniak established a classification as a tool for the characterization of cystic renal lesions detected by contrast-enhanced computed tomography (CECT), and this has become the gold standard [3]. The detection of cystic renal lesions has dramatically increased with the continued growth in cross-sectional imaging, and these lesions are often incidental findings in asymptomatic patients. B-mode ultrasound (US) can reliably categorize cystic renal lesions as simple or complex cysts. The introduction of ultrasound contrast agents (UCA) has significantly improved the capability of US to further evaluate any indeterminate lesion allowing definitive characterization [4-6]. Ultrasound practitioners have adapted this CT-based classification when evaluating complex cystic renal lesions using UCA with some success. In the recent EFSUMB Guidelines regarding non-contrast-enhanced ultrasound (CEUS) of the liver, it was recommended to use a UCA to characterize any complex renal cysts and to apply a Bosniak categorization [4]. However, with the higher temporal and spatial resolution of CEUS compared to CECT, there is a need to adapt the Bosniak classification according to the CEUS findings [7, 8].

This position paper provides guidance for the use of CEUS for the evaluation of renal cysts, following a literature review with an expert opinion in keeping with the EFSUMB guideline policy [9].

\section{Imaging of Cystic Renal Lesions: The Challenge}

While simple renal cysts have a typical appearance on B-mode US, characterization of complex cystic lesions as benign or malignant may be problematic. Malignancy may be missed within non-tumor echogenic content and, in turn, this echogenic material may simulate the presence of malignancy. Conventional Doppler US techniques can be used to evaluate vascularity of septations and solid components, but Doppler US often fails to detect slow flow in small vessels $[10,11]$. Renal cystic lesions that are not unequivocally characterized as benign with conventional US techniques require further assessment with a contrast examination. Although CECT is used more often, there is evidence that contrast-enhanced magnetic resonance imaging (CEMRI) and CEUS are at least as effective as CECT imaging for cystic lesion characterization. Benign lesions do not demonstrate internal enhancement, while the presence of enhancing soft-tissue components is strongly predictive for malig- nancy $[10,12,13]$. Contrast-enhanced ultrasound has the advantage of being able to monitor lesion vascularity in real time for several minutes at high frame rates $[14,15]$.

POSITION STATEMENT 1

Contrast-enhanced ultrasound can be used to characterize cystic renal lesions.

\section{The Bosniak Classification System}

The classification of cystic renal lesions introduced by Bosniak for CECT in 1986 [3] and recently modified by Silvermann et al. in 2019 [16] remains pertinent to the CECT diagnosis and management of complex cystic lesions [12, 17]. Cysts are classified based on the presence of certain imaging features that determine the likelihood of malignancy including hyperdensity, septations, calcifications, wall thickening, and enhancement characteristics. A "Bosniak" score is assigned to reflect the interpretation, with an increasing likelihood of malignancy $[3,18,19]$ :

- Category I-Il: The cystic lesion is a simple or a minimally complex cyst, regarded as "clearly benign" with no further evaluation required. The prevalence of malignancy in Bosniak categories I and II is reported at 3.2\% (95\% Cl 0-6.8) and $6.0 \%$ (95\% Cl 2.7-9.3), respectively [20]. In surgically treated Bosniak category II, a malignancy rate of around $9.0 \%$ (5-14\%) is reported [21].

- Category IIF: The cystic lesion is "presumably benign" with imaging surveillance advised [17]. The malignancy rate for Bosniak category IIF is $6.7 \%$ (95\% Cl 5-8.4) [20] or $18 \%$ (12$26 \%$ in surgical cohorts [21]. During imaging surveillance, re-classification to Bosniak category III/IV was necessary in $12 \%(8-17 \%)$ with a malignancy rate as high as $85 \%(74-92 \%)$ in re-classified cystic lesions.

- Category III: The cystic lesion is "indeterminate" for malignancy. The malignancy rate in Bosniak category III is $55.1 \%$ (95\% Cl 45.7-64.5) [20], and in a surgical cohort is $51 \%$ (42$61 \%)$, and in radiological cohorts $54 \%$ (45-63\%) [21].

- Category: IV: The cystic lesion is likely malignant. The malignancy rate in Bosniak category IV is 91 \% (95\% Cl 87.7-94.2) [20], with no difference between surgical cohorts (malignancy in $86 \%$ of cases) and radiological cohorts (malignancy in $95 \%$ of cases) [21]. 
The Bosniak classification system is not intended to be used alone to guide the management of patients with complex renal cysts. Management of complex renal cystic lesions is dependent on the individual patient's combination of imaging findings, clinical factors, and available treatment options [16]. The treatment decision lies with the urologist and/or the multidisciplinary renal cancer teams, based on current clinical practice and scientific evidence provided by urological guidelines [22]

\section{POSITION STATEMENT 2}

To guide the management of patients with renal cystic lesions, the Bosniak classification of imaging findings should always be used in conjunction with the assessment of clinical data and individual treatment options. irregularity and intense mural enhancement is a strong predictor of malignancy [31]. Magnetic resonance imaging led to category migration with a change in the management of complex renal cysts in a significant proportion of cases; upgrades with MR imaging in $40 \%$ [32], $23 \%$ [28], or $10 \%$ [27]. An inherent artifact with MR imaging is the depiction of thicker septa than on CECT [17, 25, 32,33 . Diffusion-weighted MRI (DWI) can provide additional information (on the presence of a tumor tissue component that may help differentiate certain cases of complex renal cysts from cystic carcinomas) [34].

\section{POSITION STATEMENT 4}

CEMRI and CECT have similar accuracy in the evaluation of renal cystic lesions using the Bosniak classification in the majority of cases.

\section{How good is the interobserver agreement between $\mathrm{CT}$, CEUS, and MR imaging classification of renal cystic lesions?}

The crucial distinction is between Bosniak categories IIF and III, as category IIF may be followed-up but, in the case of category III, surgery is often indicated. The reported overall sensitivity of the CECT Bosniak cyst classification is $93 \%$ (95\% Cl 89-95) with a specificity of $67 \%$ (95\% Cl 59-76) [20]. Magnetic resonance imaging is superior to CECT in identifying lesion septations and enhancement, resulting in a higher category, most often upgrading from Bosniak category II and IIF to IIF and III, respectively [23-25]. There is reported discrepancy regarding the performance among reporting radiologists in categorization. This discordance was greatest in the challenging categories II and III. The introduction of category IIF reduced this difficulty $[24,26]$. A recent refinement of the CECT and CEMRI criteria may be helpful but requires validation [16].

\section{POSITION STATEMENT 3}

Risk assessment of renal cystic lesions using the CT-based Bosniak classification can be challenging and is subject to interobserver variability.

\section{How accurate is risk assessment of renal cystic lesions using the MR-based Bosniak classification?}

Both MR and CT imaging have similar results in the evaluation of the Bosniak categories $[27,28]$. The pooled sensitivity and specificity for MR imaging were 0.92 and 0.91 , respectively, with an AUROC of $94.7 \%$ [29]. Using enhancement subtraction imaging, the sensitivity was improved to $95 \%$ [30], and combined mural
There was excellent interobserver agreement for Bosniak classification for both CECT (kappa score $k=0.87)$ and CEMRI $(k=0.93)$ between two readers [21]. Conversely, there was considerable disagreement among three radiologists for CECT [21, 26]. Comparing $C T$, MR, and CEUS imaging, there was agreement between CT and MR imaging in $78 \%(\mathrm{k}=0.91)$ of the cases and agreement between CT and CEUS imaging in $79 \%(\mathrm{k}=0.86)$ with discordance only in Bosniak classes II and IIF [25].

\section{POSITION STATEMENT 5}

Considerable interobserver variability in Bosniak classification of renal cystic lesions with CECT exists which may have a significant impact on clinical decision making.

\section{Ultrasound Examination of Bosniak Cysts: Contrast Agents and Dose Administered}

Different UCAs have been used in different studies, with no comparative studies being published. The greatest experience is with the sulfur hexafluoride-filled microbubble SonoVue (Bracco SpA, Italy), the agent used almost exclusively in Europe for CEUS of abdominal organs [4, 35]. The recommended dose for intravenous use of SonoVue in renal cyst characterization ranges between 0.6 to $2.4 \mathrm{~mL}$, but depends on the US system and patient habitus [36]. If needed, a second dose of the UCA may be safely administered to reexamine the kidney or for further examination of the contralateral kidney. 


\section{POSITION STATEMENT 6}

Different ultrasound contrast agents can be used to evaluate renal cystic lesions with CEUS, with sulfur hexafluoride-filled microbubbles being the agent with the greatest amount of documentation regarding efficacy.

\section{Ultrasound Examination of Bosniak Cysts: Contrast Agent Safety}

Ultrasound contrast agents are administered safely in various applications with minimal risk to patients [37-40]. The risk of an anaphylactoid reaction is low (1:7000 patients, $0.014 \%)$ and significantly lower compared to iodinated CT contrast agents (35-95:100 000 patients, 0.035-0.095\%), and comparable to the rate of severe anaphylactoid reactions associated with gadolinium-based contrast agents at $0.001-0.010 \%$. Serious anaphylactoid reactions to UCAs are observed in approximately 1:10 000 exposures. In most cases allergy-like events and hypotension occurred within a few minutes following the injection of the UCA [37, 41-43]. Due to the fact that UCAs are not excreted through the kidneys, there is no need for renal function blood tests prior to UCA injection [44, 45]. There is no evidence of any effect on renal function. Patients with renal insufficiency have no risk of contrastrelated nephropathy. Most observed adverse events were mild and resolved spontaneously within a short time without sequelae. The safety profile in children reflects that in adults $[46,47]$.

\section{POSITION STATEMENT 7}

Microbubble contrast agents for ultrasound imaging are safe and should be considered particularly in children and in patients with renal insufficiency.

\section{Ultrasound Examination of Bosniak Cysts: Equipment}

Successful CEUS examinations require use of high performance contrast-specific software, which enables separate processing of non-linear microbubble signals and linear signals emitted by normal tissue. A low mechanical index (MI) should be used in order to minimize non-linear soft tissue signals and to avoid unintentional microbubble destruction. Generally, a low MI examination is typically considered $<0.3$ not only to minimize microbubble disruption, but also to reduce tissue harmonics and artifacts. The optimum MI values vary with the different US manufacturers. Modern US machines can display a real-time dualscreen view, comprising a CEUS image alongside the B-mode US image. Particularly, this is helpful for the CEUS investigation of smaller lesions. Importantly, the B-mode US image is formed using low $\mathrm{MI}$ and is of inferior quality compared to the normally used B-mode image. Additionally, some equipment provides the possibility of a single screen presentation mode, displaying the CEUS image in an overlay mode together with the B-mode US image.

\section{POSITION STATEMENT 8}

Use of ultrasound equipment with high-performance contrast-specific modes is essential for CEUS investigation of renal cystic lesions.

\section{Ultrasound Examination of Bosniak Cysts: Investigator Training}

In order to ensure high quality of CEUS imaging, EFSUMB suggests that CEUS should be performed by practitioners with at least competence level 1 (preferably level 2 for the kidneys), as the diagnostic performance of CEUS is operator-dependent and correlates with the experience of the operator [48-50]. Additionally, physicians should ensure that their US machine is configured for adequate CEUS imaging and data post-processing. Familiarity with administration of the available UCA as well as knowledge of potential contraindications and side effects is mandatory. The operator must also be aware of the local national medico-legal regulations.

\section{POSITION STATEMENT 9}

The diagnostic performance of CEUS depends on the competence and skill of the examiner.

\section{Ultrasound Examination of Bosniak Cysts: Examination Techniques}

The renal cystic lesion may have been found on a routine US examination, or have been seen on CT without adequate characterization, for instance during a CT examination for possible renal colic. A curved array transducer, with a frequency between 1 and $9 \mathrm{MHz}$ is deployed although linear transducers with a higher frequency can also be used in the detection of superficial renal cysts [51]. Following B-mode US to identify lesion location and color Doppler US to assess vascularity, the best approach to perform the CEUS examination is determined. Prior to the administration of the UCA, it is good practice to get oral or written informed consent for the use of intravenous contrast agents, according to local regulations. The examination should be performed with both the patient and the examiner in a comfortable position, with a view of the lesion in a longitudinal plane to allow continuous observation during respiration.

The UCA is administered by an assistant, and the examination is recorded continuously for at least 60 seconds and still images thereafter [52]. The kidneys enhance rapidly and intensely after UCA administration, with potential to assess both the macroand the microvasculature, the former immediately after UCA arri- 
val. The arterial pedicle and main arterial branches enhance first, followed rapidly by the segmental, interlobar, arcuate and interlobular arteries and then complete cortical enhancement. Medullary enhancement follows, with the outer medulla enhancing first, followed by gradual fill-in of the pyramids [53]. As UCAs are not excreted by the kidneys, there is no UCA in the renal collecting system. With CEUS only two enhancement phases occur: a cortical phase, 15-30s after UCA administration with cortical enhancement seen, and a parenchymal phase, when both the cortex and medulla enhance at $25 \mathrm{~s}-4$ mins after UCA administration. There is normally excellent depiction of renal perfusion throughout the kidney, superior to color Doppler US techniques. Contrast enhancement is reported to be less intense and fades earlier in patients with chronic renal disease [54]. Any abnormal enhancement pattern, when compared with the marked enhancement of the cortex, should be observed for subsequent wash-out, thought to be an indicator of malignancy $[4,36]$. It is important to record the examination as a dynamic cine clip, and to review the examination carefully following completion of the examination [52].

\section{POSITION STATEMENT 10}

An appropriate examination technique is important to evaluate complex renal cystic masses accurately both with conventional US modes and CEUS.

\section{POSITION STATEMENT 11}

CEUS precisely depicts renal vascularization and its changes in pathological conditions.

\section{Bosniak Cyst Classification on Multiparametric Imaging}

The Bosniak categorization is a scale of increasing probability of cancer based upon imaging features and works well for cystic renal lesion evaluation in clinical practice [55]. The CECT-based Bosniak cyst classification system has been used to categorize cystic renal lesions on CEMRI and CEUS, with comparable results [7, 8, 56-58], but both CEMRI and CEUS tend to upgrade complex renal cystic lesions [59]. Imaging methods evaluate the various aspects of renal cystic lesions in different ways, and the single features are valued with different degrees of sensitivity and specificity. This must be considered when assigning the Bosniak category based on CECT, CEMRI, or multiparametric US. In particular, a CEUS examination performs better than CECT in the detection of lesion vascularity $[59,60]$, depicts more septa and is superior in depicting the degree of both septal and wall thickening, septal enhancement and enhancement of solid components within the lesion compared with CECT $[7,56,61,62]$. CEUS is extremely sensitive in revealing even the tiny capillaries that feed hair-line thin septa with a superior temporal and spatial resolution compared to any other imaging modality [63], with the potential to falsely upgrade lesions when applying the original Bosniak criteria for categorization [8, 24, 56, 61-66]. Contrast-enhanced ultrasound inherently demonstrates more complexity in cystic lesions and has the potential to improve lesion characterization and change therapeutic management effectively [5, 14]. Cystic renal lesions initially categorized on CECT can be subject to CEUS to improve diagnostic accuracy $[67,68]$. Modified or new diagnostic Bosniak categorizations for CEUS, to improve specificity and overall performance, have been advocated but these are inconsistent $[8,14,15,63,68]$. Most of the investigators who use a CEUS-modified Bosniak category actually use the Bosniak scoring system but assign the Bosniak scores through imaging criteria specific for CEUS. Any modified categorization using CEUS should define these criteria unequivocally, rather than developing a separate classification.

\section{POSITION STATEMENT 12}

Caution should be used when applying the criteria developed for CECT to CEUS as the criteria for Bosniak categorization vary depending on which imaging technique is used.

\section{POSITION STATEMENT 13}

Medical reports must state which imaging technique was used to classify a particular renal cystic lesion.

\section{Bosniak Cyst Classification: Unique Features of CEUS}

The key features to be considered are the presence of enhancing wall and septa, with or without irregularities, and intralesional enhancing masses or nodules. Areas of calcification pose a difficulty for imaging with US and sometimes with MRI and CT, interfering with the assessment of enhancement [69]. Time intensity curve analysis of an administered contrast agent has no established role for the classification of renal cystic lesions [36, 61]. The characteristics of contrast enhancement on CEUS and CECT are different. The UCA agent is strictly intra-vascular, while agents used in CEMR and CECT have an equilibrium phase in which contrast leaks out of vessels. Therefore, the criteria used to score the lesions on CECT must be adapted to the CEUS technique.

These are the most relevant differences between CEUS and CECT:

- Attenuation is a specific criterion for CECT scanning. The presence of echogenic content can act as a surrogate for high attenuation [15], although it is not equivalent, since hyperdense cysts can show anechoic content on B-mode US [12, 60].

- CEUS cannot differentiate between perceived and measurable enhancement, as enhancement is either present or not. Of note, perceived enhancement is no longer considered in the current CECT/CEMRI categorization [16]. A surrogate could be the identification of single microbubbles running within tiny 
- Table 1 Bosniak renal cyst classification on multiparametric US.

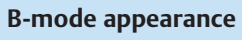

Cysts with internal debris, echogenic content, or mixed appearance

\section{CEUS appearance}

Thin wall without irregularities that show no enhancement on CEUS, or individual microbubbles running within tiny vessels in the wall

CEUS not

CEUS not

necessary

Thin wall and septa without irregularities showing no enhancement, or individual microbubbles running within tiny vessels in the wall and septa

Bosniak score on multiparametric US

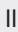

II

Thin wall and septa without irregularities showing no enhancement, or individual microbubbles running within tiny vessels in the wall and septa

Cysts with multiple septa, internal debris, echogenic content, or mixed appearance. Calcifications of the wall and/or septa may be present slightly hampering the evaluation of the cyst wall, content, and septa

Totally intrarenal cysts otherwise meeting the category II criteria

CEUS necessary

Cysts with multiple septa, internal debris, echogenic content, or mixed appearance

CEUS necessary

Cysts with multiple septa, internal debris, echogenic content, or mixed appearance

\section{CEUS} necessary
Multiple septa, thin or minimally thickened

(2-3 mm). Smooth or minimally thickened wall vessels in the wall and septa, a phenomenon which is believed to be responsible for perceived enhancement [12].

- CEUS is superior to CECT in detecting enhancement. Septa can appear thicker, and subtle wall/septa irregularities are more evident on CEUS. Moreover, thin septa with faint enhancement can appear thicker and with heavy enhancement if an excessive dose of UCA is injected (microbubble piling and blooming artifact).

- The presence of cyst wall calcification, with acoustic shadowing, may hamper the visualization of any deeper enhancing nodules or septa, making lesion categorization ineffective [70].

- Large patient habitus or overlying bowel gas may also obscure visualization with US.

- Nodules are only seen in Bosniak IV complex renal cysts and are easily distinguished from localized wall or septal thickening on a CEUS examination.

\section{Bosniak Cyst Classification: Scoring Criteria on Multiparametric US}

The criteria for a US-based Bosniak category assessment have been reported with notable differences from the CT-based cate- gories $[6,15,51,61,70-72]$. While characterization of simple cysts (category I) and of a subgroup of minimally complicated benign cysts (category II) is obtained on B-mode US, the majority of complex renal cysts are effectively characterized on CEUS. The criteria described below represent a synthesis of those reported in the different studies. - Table 1 shows how Bosniak scoring is obtained using multiparametric US, following the recommendations of the present paper.

- Category I: Simple benign cysts. These cysts meet the sonographic criteria for simple cysts anywhere in the body: thin $(<2 \mathrm{~mm})$ wall, sharp margins without irregularities and calcifications; anechoic content; posterior acoustic enhancement [68]. These lesions are fully characterized as benign on B-mode US; no UCA administration is needed ( $\triangleright$ Fig. 1).

- Category II: Minimally complex benign cysts. These cysts present with one of the following appearances: Cysts that meet the criteria of simple cysts, but with a few [1-3] thin $(<2 \mathrm{~mm})$ septa without irregularities [68]. Calcification of the wall and/ or septa may be present which do not hamper evaluation of the cystic content. These lesions are characterized as benign on B-mode US ( $\triangleright$ Fig. 2). No UCA administration is needed, but, if used, individual microbubbles are demonstrated within 

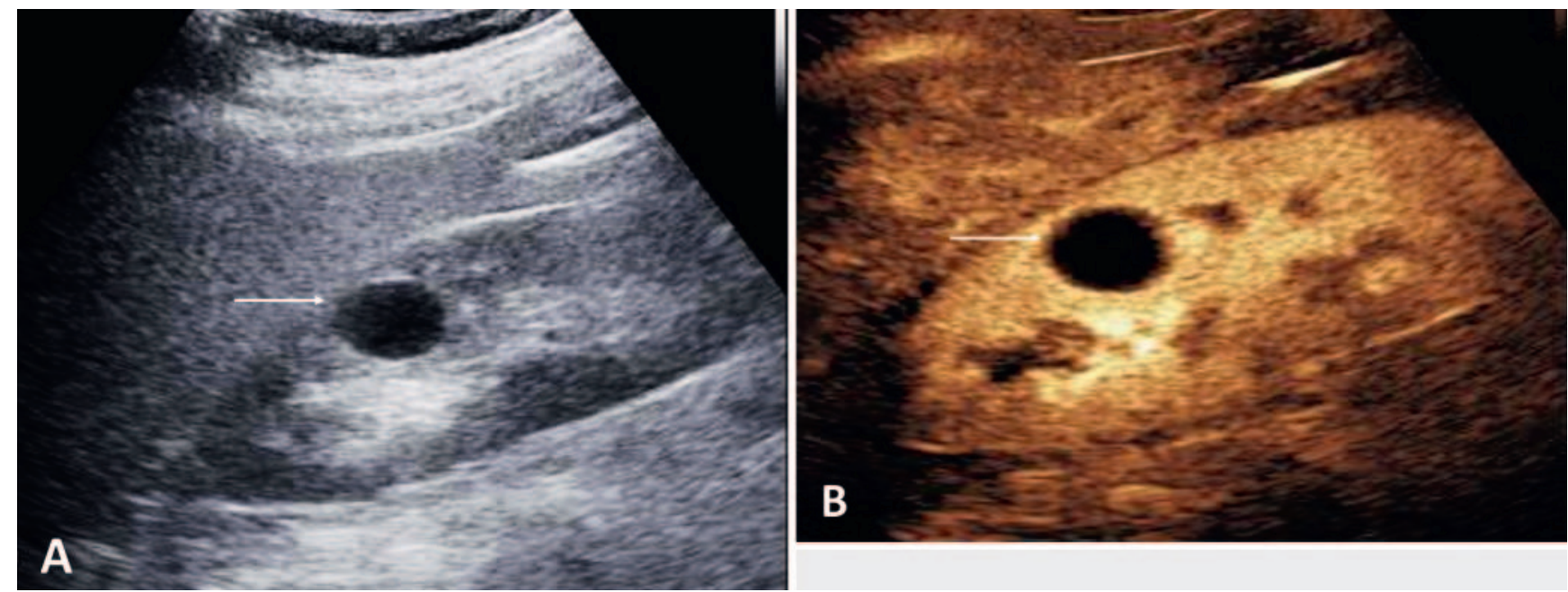

- Fig. 1 Bosniak category I cyst. A The cyst (arrow) shows anechoic content with posterior acoustic enhancement, a thin ( $<2$ mm) border with sharp margins, no irregularities, calcifications, or septa. Simple cysts are fully characterized as benign on B-mode US. B CEUS is not needed to confirm the findings, but if an incidental cyst is encountered during an examination for a separate renal lesion, the findings on B-mode US is confirmed.
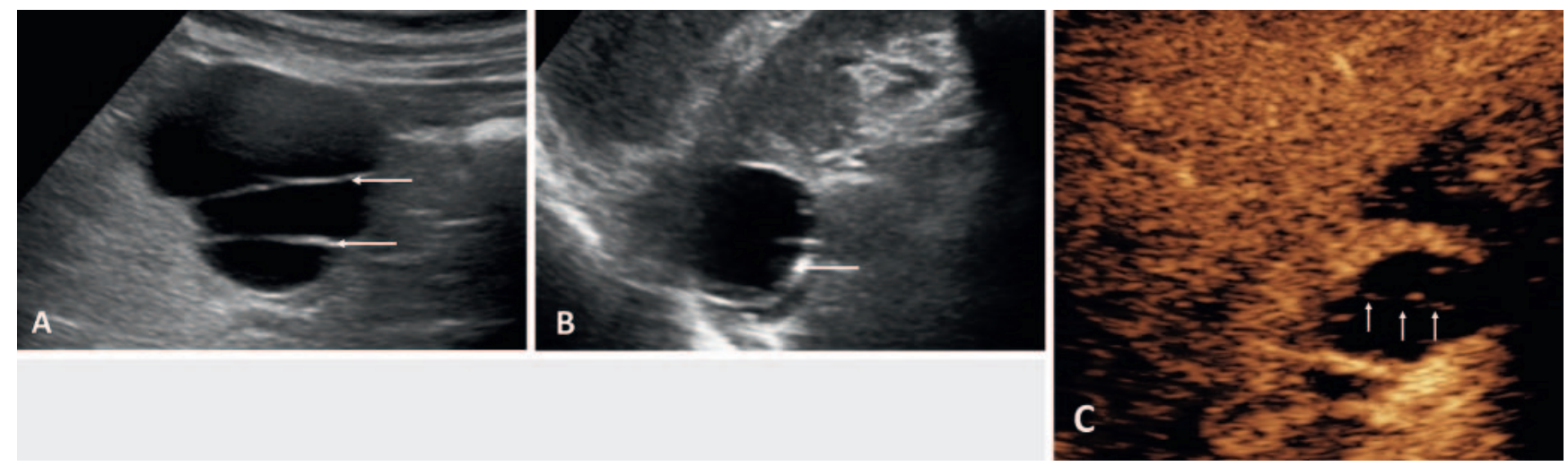

- Fig. 2 Bosniak category II cyst A Minimally complex benign cyst displaying 2 thin septa (arrows) without irregularities. Category II cyst not requiring further investigation on CEUS. B Cyst displaying thin wall calcifications (arrow). Category II cyst not requiring further investigation on CEUS. C CEUS is not needed to confirm the findings, but if an incidental septated cyst is encountered during an examination for a separate renal lesion, the vascularization of the septa (arrow) is clearly identified as thin enhancing linear areas within the cyst.

tiny vessels in the wall and septa $[6,7,73]$. Cysts with internal debris, echogenic content, or a mixed appearance with thin wall that show no enhancement on CEUS ( $\triangleright$ Fig. 3), a limited number of thin septa [1-3] without irregularities, or few microbubbles identified in the wall or septa [36].

- Category IIF - Presumably benign, imaging surveillance is advised. Cysts with multiple thin septa, minimally thickened (2$3 \mathrm{~mm}$ ) smooth septa and cyst border ( $\bullet$ Fig. 4). Internal debris, echogenic or mixed content, and calcification may be present $[6,58,68,73]$. Cysts meeting the category II criteria with existing calcification slightly hampering the evaluation of the cyst wall, content, and septa. Totally intrarenal cysts otherwise meeting the category II criteria for which differentiation between non-enhancing and enhancing border cannot be achieved ( $\triangleright$ Fig. 4C) [15].
- Category III - Indeterminate lesions. Cystic lesions with enhancing smooth thick ( $\geq 4 \mathrm{~mm}$ ) wall or septa, and/or with enhancing irregular (>3 mm) walls and/or septa ( $\triangleright$ Fig. 5). No nodules are seen $[6,15,36,58,68,70,73]$.

- Category IV - Likely malignant cystic tumors. Cystic lesions with the characteristics of category III cysts, which also contain enhancing soft-tissue protrusions ( $\triangleright$ Fig. 6), either nodules with obtuse margins ( $\geq 4 \mathrm{~mm}$ ), or with acute margins of any size $[6,15,36,63,70,73]$.

\section{POSITION STATEMENT 14}

The likelihood of malignancy of complex renal cystic lesions can be assessed using CEUS-based criteria. 

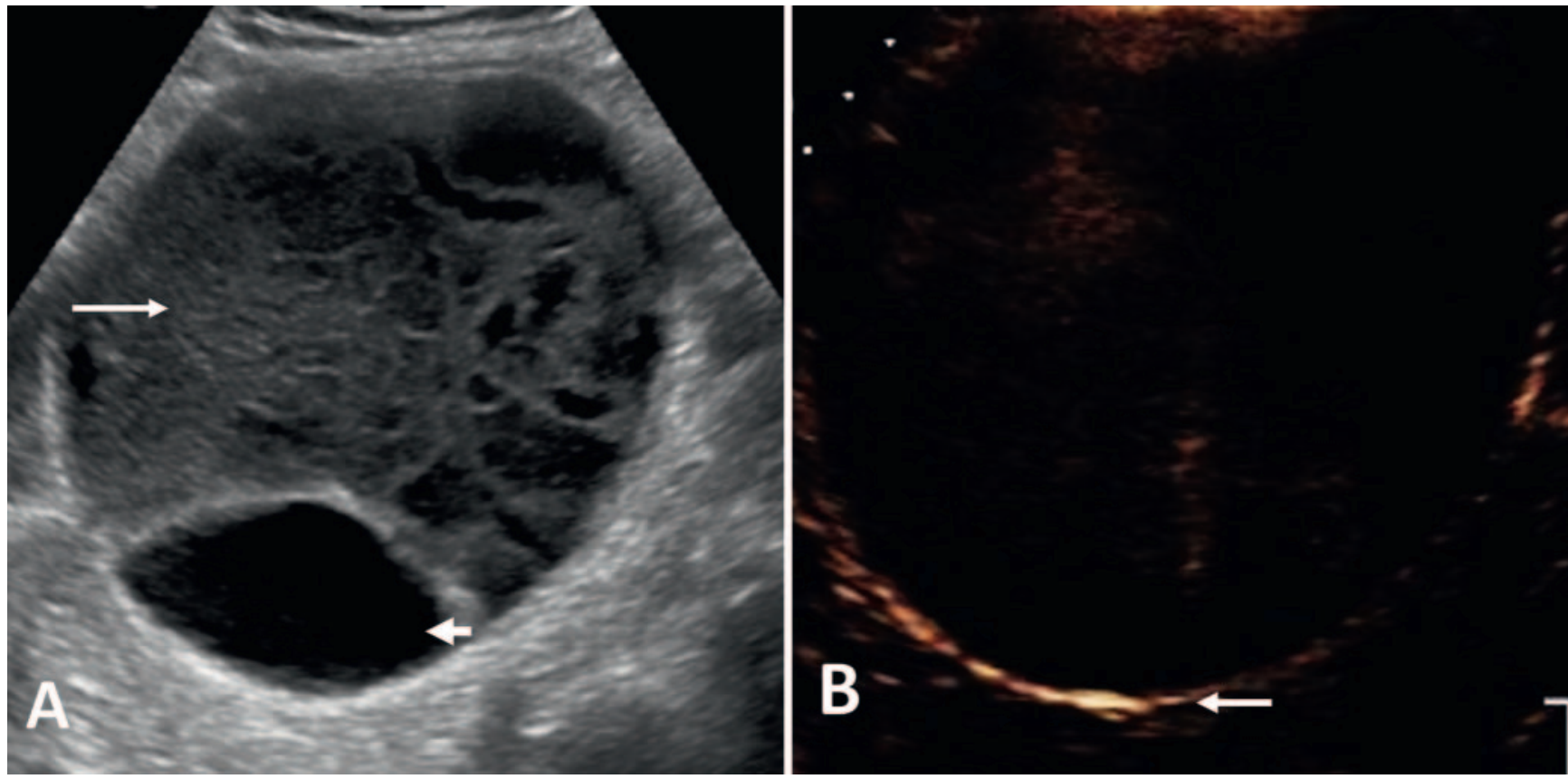

- Fig. 3 Bosniak category IIF cyst A B-mode US shows a lesion with mixed appearance, with both a solid (arrow) and cystic component (arrowhead). B CEUS examination demonstrates no enhancement within the lesion, a thin wall without irregularities (arrow), and no septa.
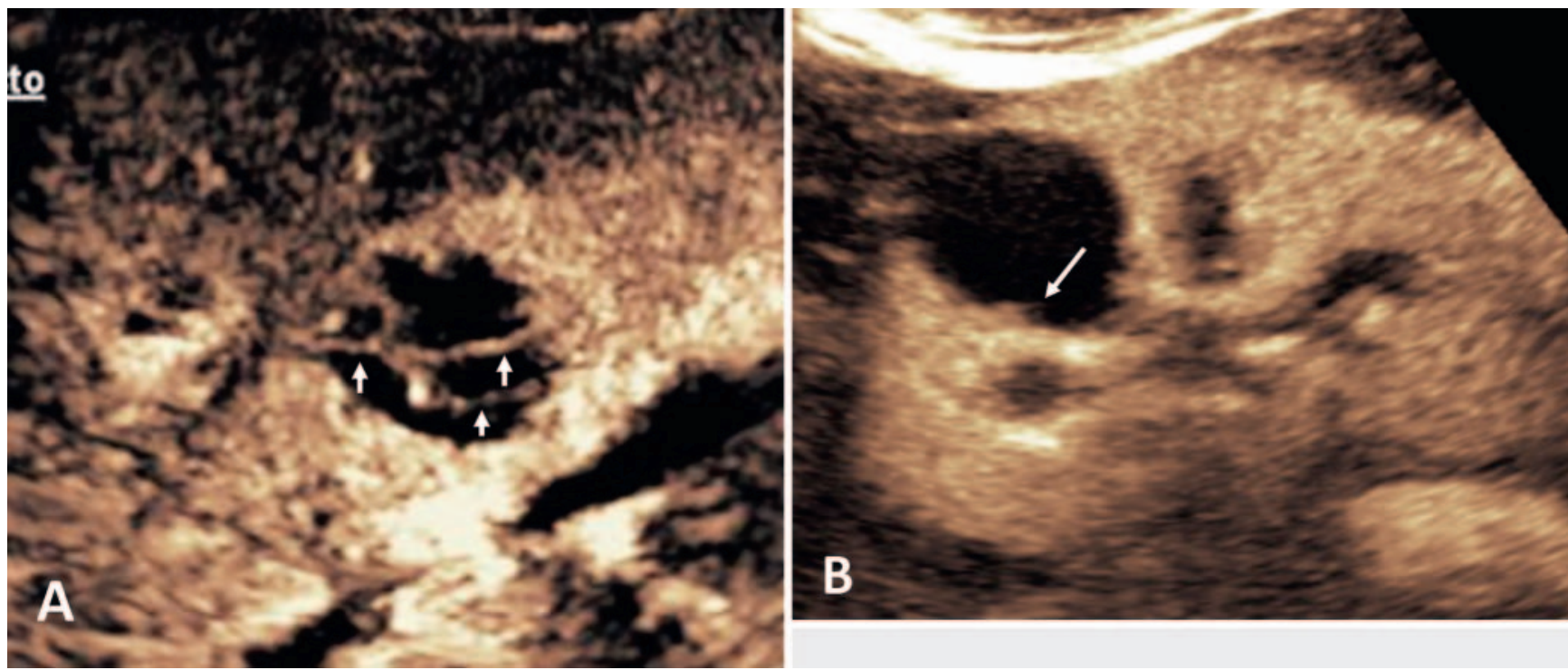

- Fig. 4 Bosniak category IIF cyst. A CEUS examination of an intrarenal cyst with multiple, minimally thickened, enhancing septa (arrows). B CEUS examination of an intrarenal cyst with a focal thickening $(2 \mathrm{~mm})$ of the wall (arrow).

\section{Specific points regarding the CEUS Bosniak classification}

Cysts with thick or nodular calcifications, without hampering evaluation of cyst content, are placed in category IIF; calcification is not a sign of malignancy provided that there is no associated suspicious lesion [18, 51, 73]. There is no definition for a threshold for labelling a septum as 'thick' [32]; septa $\leq 1 \mathrm{~mm}$ are considered thin, by the majority of investigators [6, 62, 70, 73]. Others suggest a $2 \mathrm{~mm}$ threshold $[8,51,72,74]$. A "hairline septum" is a subjective assessment, dependent on the US equipment used, for which a precise thickness threshold cannot be assigned effectively. There is no threshold to differentiate between "few septa" and "multiple septa" with an arbitrary threshold at three septa. "Few" equals $1-3$ and "many" is $\geq 4$ septa $[16,32,75]$. Lesion size is not a consideration for cyst categorization with conflicting results for predicting malignancy [76-80]. On CECT, totally intrarenal non-enhancing high-attenuation cysts $<3 \mathrm{~cm}$ are assigned to category II, while cysts with the same characteristics $>3 \mathrm{~cm}$ are assigned to category IIF [12]. Large $(\geq 3 \mathrm{~cm})$ homogeneous, 


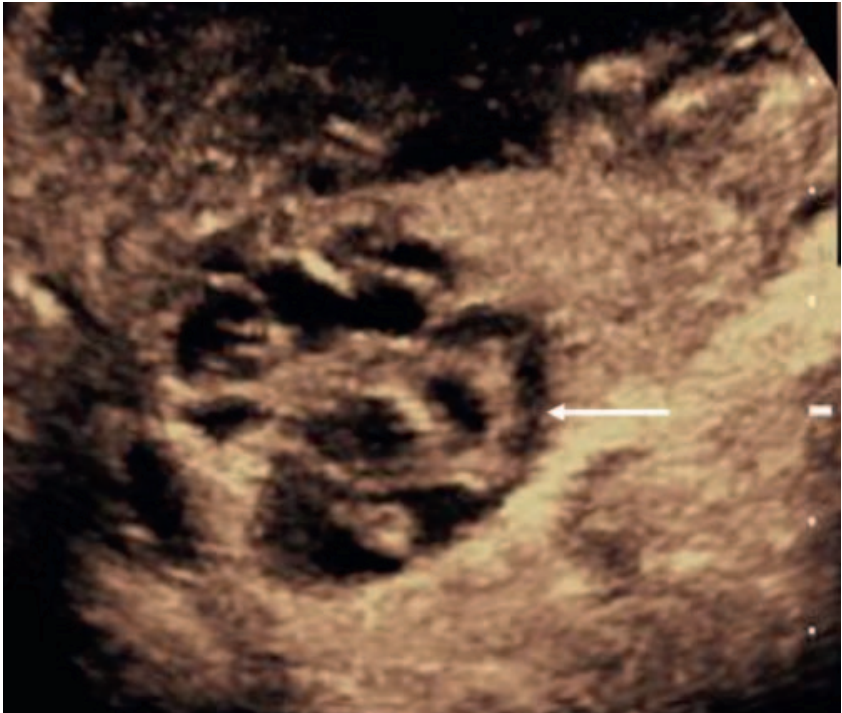

- Fig. 5 Bosniak category III cyst. A CEUS examination of an indeterminate cystic lesion with the presence of thick enhancing wall and septa.

hyperattenuating, non-enhancing renal masses, originally considered IIF masses, are rare. The need for follow-up is because of difficulty in the differentiation between non-enhancing and enhancing wall.

\section{POSITION STATEMENT 15}

The features of US modes must be taken into consideration when criteria developed for CECT and for CEMRI are adapted to categorize renal cystic lesions on CEUS.

\section{Reporting the CEUS Bosniak classification}

A CEUS report should include information on examination conditions, quality, and limitations. All technical limitations that could hamper a confident diagnosis should be detailed, such as: difficult-to-image patient, deep position of the cyst, calcification hampering evaluation of the intra-cystic content, large cysts incompletely examined due to superimposition of bowel gas, poor acoustic window or other barriers, and presence of artifacts. Lesion appearance on B-mode US should be reported, in particular, with regard to the presence of septa, wall and septa calcification, presence of echogenic cyst content, and features on color Doppler techniques. Then, lesion appearance on CEUS should be described with an emphasis on the presence of smooth enhancing wall and septa, enhancing wall and septa irregularities (either circumscribed or diffuse, subtle or marked), and on presence of enhancing solid components. The intensity of observed enhancement should be subjectively described either as unequivocal enhancement, or identification of single microbubbles running within tiny vessels in the wall and septa. A cine clip should be recorded for subsequent evaluation of the CEUS examination.

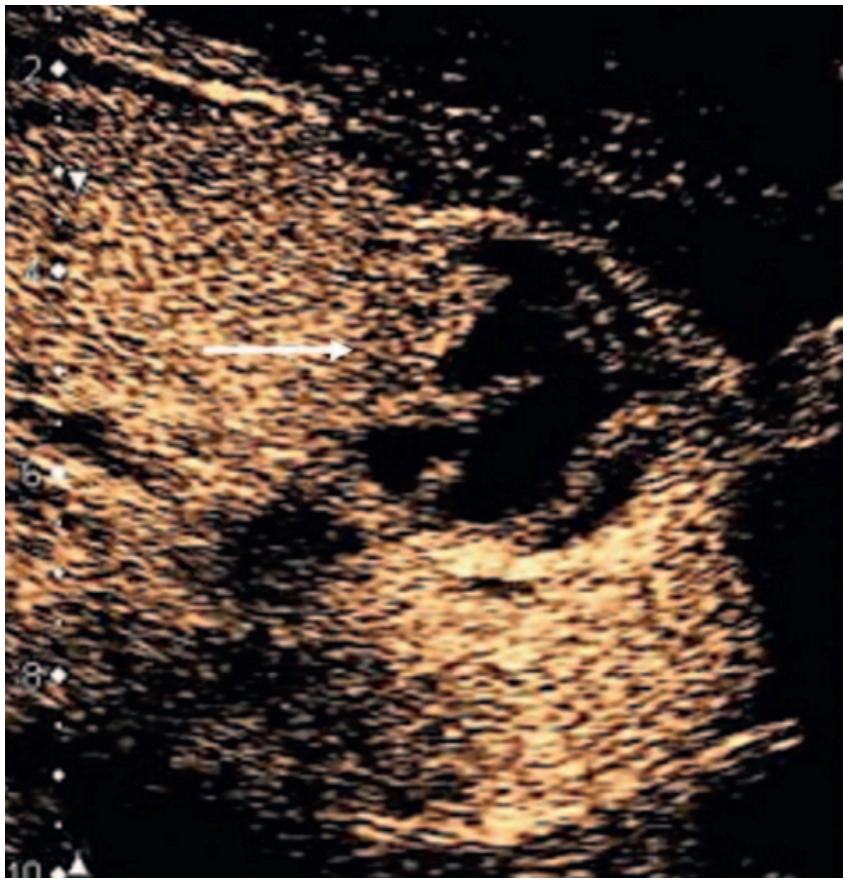

- Fig. 6 Bosniak category IV cyst. A CEUS examination indicating a likely malignant cystic lesion with the presence of thick enhancing septa and a wall vegetation (arrow).

\section{POSITION STATEMENT 16}

A comprehensive description of the appearance on grayscale, color Doppler US, and CEUS cine clips should be reported when scoring a renal cystic lesion according to the Bosniak criteria.

\section{Limitations of Bosniak scoring on CEUS}

Complex cystic renal masses pose a particular interpretative challenge for the observer because the imaging boundary between benign and malignant lesions is often unclear. The Bosniak classification works well but is intrinsically subjective when applied to other imaging techniques and is dependent on the observer's experience. This leads to an unavoidable high degree of interobserver disagreement [65]. Moreover, the main limitations of B-mode and Doppler US will apply to CEUS as well. Heavily calcified lesions cannot be evaluated. Assessment is influenced by the location of the lesion in poorly visualized kidneys, shadowing from bowel gas or ribs, and patient body habitus.

\section{POSITION STATEMENT 17}

When scoring renal cystic lesions on CEUS the intrinsic limitation of this technique must be considered. 


\section{Controversies with CEUS of Bosniak Categories}

\section{Does the CEUS-based classification upgrade or downgrade the risk of malignancy of renal cystic lesions?}

The presence of enhancement, indicating neovascularization, is the most important factor in determining the need for surgery in cystic renal lesions $[12,13]$. Improved CT resolution when compared to the original CECT categories has resulted in fewer indeterminate cyst findings and increased specificity [81]. With CEUS, $31 \%$ of renal cysts were attributed a higher Bosniak category compared to CECT [7, 8, 13, 56, 65]. The increased contrast enhancement and better temporal and spatial resolution of CEUS (and MR imaging) demonstrate previously undetected features. Minimal septa enhancement is not indicative of malignancy, and an increased sensitivity of CEUS demonstrating enhancing nodules not seen with CECT has been noted [56, 82]. A similar higher Bosniak category with MR imaging has been seen [10, 28, 32] but an apparent wall thickening artifact is an issue [66]. Both upgrading and downgrading of Bosniak categories with MRI and CEUS compared to CT imaging is apparent in $>20 \%$ of cases [25], with CEUS demonstrating lower specificity but improved sensitivity and accuracy compared to MR imaging [57].

\section{POSITION STATEMENT 18}

The imaging criteria used to assign the Bosniak categories are developed for CECT and must be adapted to be successfully applied for scoring on CEMRI and CEUS, as changes in category will occur.

\section{Should CEUS be considered an equivalent, complimentary, or alternative technique to contrast- enhanced CT for renal cystic lesions?}

Although CECT is the reference standard for Bosniak categories of renal cystic malignancy risk, CECT is inherently inaccurate, with a reported sensitivity of $89.6 \%$ and specificity of $65.1 \%$ in distinguishing between benign and malignant renal cysts [20]. The comparability of CEUS with the reference standard of CECT has been addressed with excellent agreement $[7,56,83]$ with a single study indicating that experience in CEUS interpretation is crucial [65]. There was a potential for CEUS to overestimate the Bosniak category, with the 'real-time' examination able to demonstrate minor enhancement (a marker of malignant potential). The current view suggests that this is an advantage, rather than a drawback. This requires, however, a fundamental change in imaging assessment of renal cysts, centered on CEUS demonstration of lesion vascularity [5]. When CEUS is inconclusive due to poor visualization (i. e., due to patient habitus or poor acoustic window), CECT usually permits better characterization and furthermore allows staging of a malignant renal lesion. There is better demonstration of calcification on CECT which could affect the Bosniak category on US $[18,84]$. Using CEUS only in cases of contraindications or non-acceptance of CECT is not justified based on the current knowledge of the potential of this technique and would be detrimental to acquiring further cumulative experience in CEUS.

\section{Characteristics of non-progressive Bosniak Category IIF cysts}

The initial cyst size, change in lesion size (increase or decrease), and growth rate [growth rate $=$ (follow-up size minus initial size)/ years between measurements] were not found to correlate with progression. A multilobulated border of the lesion was not found to correlate with progression and no lesions with calcification progressed. Growth rates in cystic lesions are often a consequence of fluid accumulation (downgrading to a Bosniak category II). Progression to malignancy is based on the appearance of enhancing solid portions, an increase in number, thickness or irregularity of enhancing septa, and on an increase in thickness of the enhancing wall [85]. There is no difference in progression to malignancy on follow-up CECT imaging compared with MR imaging [16, 86-88]. When there is indeterminate enhancement on CECT, CEMR or CEUS imaging can be the next imaging stage $[59,89,90]$.

\section{POSITION STATEMENT 19}

Follow-up of cystic renal lesions can be carried out effectively with CECT, CEMR, or CEUS imaging. The current evidence shows similar performance for the three techniques.

\section{When should CEUS be supplemented by CT or MR imaging for follow-up?}

A CEUS examination is suited for the follow-up of nonsurgical lesions to detect any morphologic changes such as thickening of septa, appearance of a solid nodule, or contrast-enhanced alterations indicative of progression of the disease. A CEUS examination has at least the same diagnostic accuracy as CECT for renal cyst categorization but image acquisition is influenced by the location of the lesion in poorly visualized kidneys, shadowing from bowel gas or ribs, patient's constitution, and wall calcification [29, 57, 91]. Smaller lesions localized within the renal parenchyma may be difficult to characterize with CEUS, as these lesions often disappear ('masked') during a CEUS examination due to the prominent vascularity of the renal cortex, with the possibility of a lower dose of UCA being helpful. With these issues, further CT or MR imaging is necessary [25].

\section{POSITION STATEMENT 20}

CEUS-based Bosniak categorization must be supplemented by $\mathrm{CT}$ or MR imaging when there is inadequate visualization of the cystic renal lesion. 


\section{Conclusion}

The Bosniak categorization was originally formulated for CECT, and then applied to MR imaging and CEUS without adapting the criteria developed primarily for CT. With differences in imaging specifics, an inaccurate assignment of the Bosniak category and ultimately inappropriate treatment could result from the CEUS and MR imaging interpretation. The criteria used to assign the Bosniak category scores on CEUS have been reviewed, redefined, and standardized, taking into account the unique characteristics of the CEUS examination. The aim is to allow for clearly defined criteria allowing for a better assessment of the performance of CEUS in the categorization of complex renal cysts. The CEUS categorization is not intended to replace, but rather to complement the current Bosniak CT categorization, thereby improving its accuracy in the assessment of malignancy in each category. The Bosniak categorization system is used worldwide and provides a common language but is intrinsically subjective, a shortcoming which will likely be reduced when using the redefined scoring criteria which include a CEUS examination.

\section{Conflict of interest}

Vito Cantisani: lecturer fees from Bracco, Samsung, Toshiba.

Paul Sidhu: lecture fees from Bracco.

Adrian Saftoiu: lecture fees from Bracco.

Dirk-André Clevert: speaker bureau Bracco, Siemens, Esaote, Samsung, Philips.

\section{Acknowledgements}

We would like to thank Dr D. Fresilli for his help during all the phases related to the submission.

\section{References}

[1] Parienty RA, Pradel J, Parienty I. Cystic renal cancers: CT characteristics. Radiology 1985; 157: 741-744

[2] Warren KS, McFarlane J. The Bosniak classification of renal cystic masses. BJU International 2005; 95: 939-942

[3] Bosniak MA. The current radiological approach to renal cysts. Radiology 1986; 158: 1-10

[4] Sidhu PS, Cantisani V, Dietrich CF et al. The EFSUMB Guidelines and Recommendations for the Clinical Practice of Contrast-Enhanced Ultrasound (CEUS) in Non-Hepatic Applications: Update 2017 (Short Version). Ultraschall in Med 2018; 39: 154-180

[5] Barr RG, Peterson C, Hindi A. Evaluation of indeterminate renal massess with contras-enhanced US: a diagnostic performance study. Radiology 2014; 271: 133-142

[6] Rubenthaler J, Bogner F, Reiser M et al. Contrast-Enhanced Ultrasound (CEUS) of the Kidneys by Using the Bosniak Classification. Ultraschall in Med 2017; 37: 234-251

[7] Ascenti G, Mazziotti S, Zimbaro G et al. Complex Cystic Renal Masses: Characterization with Contrast-enhanced US. Radiology 2007; 243: 158165

[8] Quaia E, Bertolotto M, Cioffi V et al. Comparison of Contrast-Enhanced Sonography with Unenhanced Sonography and Contrast-Enhanced CT in the Diagnosis of Malignancy in Complex Cystic Renal Masses. Am J Roentgenol 2008; 191: 1239-1249
[9] Jenssen C, Gilja OH, Serra AL et al. European Federation of Societies for Ultrasound in Medicine and Biology (EFSUMB) Policy Document Development Strategy: Clinical Practice Guidelines, Position Statements and Technological Reviews. Ultrasound Int Open 2019; 05: E2-E10

[10] Hindman NM. Imaging of Cystic Renal Masses. Urologic Clinics of North America 2018; 45: 331-349

[11] Kazmierski B, Deurdulian C, Tchelepi H et al. Applications of contrastenhanced ultrasound in the kidney. Abdominal Radiology 2017; 43: 880-898

[12] Israel GM, Bosniak MA. An update of the Bosniak renal cyst classification system. Urology 2005; 66: 484-488

[13] Robbin ML, Lockhart ME, Barr RG. Renal imaging with ultrasound contrast: current status. Radiologic Clinics of North America 2003; 41: 963 978

[14] Barr RG. Is there a need to modify the Bosnaik renal mass classification with the addition of contrast-enhanced sonography? J Ultrasound Med 2017; 36: 865-868

[15] Chang EH, Chong WK, Kasoji SK et al. Diagnostic accuracy of contrastenhanced ultrasound for characterization of kidney lesions in patients with and without chronic kidney disease. BMC Nephrol 2017; 18: 266

[16] Silverman SG, Pedrosa I, Ellis JH et al. Bosniak Classification of Cystic Renal Masses, Version 2019: An Update Proposal and Needs Assessment. Radiology 2019; 292: 475-488

[17] Bosniak MA. The Bosniak Renal Cyst Classification: 25 Years Later. Radiology 2012; 262: 781-785

[18] Israel GM, Bosniak MA. Calcification in Cystic Renal Masses: Is It Important in Diagnosis? Radiology 2003; 226: 47

[19] Israel GM, Bosniak MA. How I Do It: Evaluating Renal Masses. Radiology 2005; 236: 441-450

[20] Sevcenco S, Spick C, Helbich TH et al. Malignancy rates and diagnostic performance of the Bosniak classification for the diagnosis of cystic renal lesions in computed tomography - a systematic review and metaanalysis. Eur Radiol 2017; 27: 2239-2247

[21] Schoots IG, Zaccai K, Hunink MG et al. Bosniak Classification for Complex Renal Cysts Reevaluated: A Systematic Review. Journal of Urology 2017; 198: $12-21$

[22] Ljungberg B, Albiges L, Abu-Ghanem Y et al. European Association of Urology Guidelines on Renal Cell Carcinoma: The 2019 Update. European Urology 2019; 75: 799-810

[23] Silverman SG, Israel GM, Herts BR et al. Management of the Incidental Renal Mass. Radiology 2008; 249: 16-31

[24] Graumann O, Osther SS, Karstoft J et al. Bosniak classification system: inter-observer and intra-observer agreement among experienced uroradiologists. Acta Radiologica 2015; 56: 374-383

[25] Graumann O, Osther SS, Karstoft ] et al. Bosniak classification system: a prospective comparison of $C T$, contrast-enhanced US, and MR for categorizing complex renal cystic masses. Acta Radiologica 2015; 57: 14091417

[26] Siegel CL, McFarland EG, Brink JA et al. CT of cystic renal masses: analysis of diagnostic performance and interobserver variation. Am J Roentgenol 1997; 169: 813-818

[27] Israel GM, Hindman N, Bosniak MA. Evaluation of Cystic Renal Masses: Comparison of CT and MR Imaging by Using the Bosniak Classification System. Radiology 2004; 231: 365-371

[28] Zhong J, Cao F, Guan X et al. Renal cyst masses (Bosniak category II-III) may be over evaluated by the Bosniak criteria based on MR findings. Medicine (Baltimore) 2017; 96: e9361

[29] Zhou L, Tang L, Yang T et al. Comparison of contrast-enhanced ultrasound with MRI in the diagnosis of complex cystic renal masses: a metaanalysis. Acta Radiologica 2018; 59: 1254-1263 
[30] Hecht EM, Israel GM, Krinsky GA et al. Renal Masses: Quantitative Analysis of Enhancement with Signal Intensity Measurements versus Qualitative Analysis of Enhancement with Image Subtraction for Diagnosing Malignancy at MR Imaging. Radiology 2004; 232: 373-378

[31] Balci NC, Semelka RC, Patt RH et al. Complex renal cysts: findings on MR imaging. Am J Roentgenol 1999; 172: 1495-1500

[32] Ferreira AM, Reis RB, Kajiwara PP et al. MRI evaluation of complex renal cysts using the Bosniak classification: a comparison to $\mathrm{CT}$. Abdominal Radiology 2016; 41: 2011-2019

[33] Weibl P, Klatte T, Kollarik B et al. Interpersonal variability and present diagnostic dilemmas in Bosniak classification system. Scandinavian Journal of Urology and Nephrology 2011; 45: 239-244

[34] Inci E, Hocaoglu E, Aydin S et al. Diffusion-weighted magnetic resonance imaging in evaluation of primary solid and cystic renal masses using the Bosniak classification. European Journal of Radiology 2012; 81: 815-820

[35] Dietrich CF, Nolsøe CP, Barr RG et al. Guidelines and Good Clinical Practice Recommendations for Contrast Enhanced Ultrasound (CEUS) in the Liver - Update 2020 - WFUMB in Cooperation with EFSUMB, AFSUMB, AIUM, and FLAUS. Ultraschall in Med 2020; 41: 562-585

[36] Harvey CJ, Alsafi A, Kuzmich S et al. Role of US contrast agents in the assessment of indeterminate solid and cystic lesions in native and transplant kidneys. Radiographics 2015; 35: 1419-1430

[37] Piscaglia F, Bolondi L. The safety of SonoVue in abdominal applications: retrospective analysis of 23188 investigations. Ultrasound in Med Biol 2006; 32: 1369-1375

[38] Main ML. Ultrasound contrast agent safety: from anecdote to evidence. JACC Cardiovasc Imaging 2009; 2: 1057-1059

[39] Main ML, Goldman JH, Grayburn PA. Thinking Outside the box: The Ultrasound Contrast Controversy. J Am Coll Cardiol 2007; 50: 24342437

[40] Tang C, Fang K, Guo Y et al. Safety of Sulfur Hexafluoride Microbubbles in Sonography of Abdominal and Superficial Organs: Retrospective Analysis of 30222 Cases. J Ultrasound Med 2017; 36: 531-538

[41] Kitzman DW, Goldman ME, Gilliam LD et al. Efficacy and safety of the novel ultrasound contrast agent perflutren (Definity) in patients with suboptimal baseline left ventricular echocardiographic images. Am J Cardiol 2000; 86: 669-674

[42] Wilson SR, Burns PN. Microbubble-enhanced US in body imaging: What role? Radiology 2010; 257: 24-39

[43] ter Haar G. Ultrasonic contrast agents: safety considerations reviewed. Eur J Radiol 2002; 41: 217-221

[44] Girometti R, Stocca E, Granata A et al. Impact of contrast-enhanced ultrasound in patients with renal function impairment. World J Radiol 2017; 28: 10-16

[45] Putz F], Erlmeier A, Wiesinger I et al. Contrast-enhanced ultrasound (CEUS) in renal imaging at an interdisciplinary ultrasound centre: Possibilities of dynamic microvascularisation and perfusion. Clin Hemorheol Microcircl 2017; 66: 293-302. doi:10.3233/CH-179103

[46] Yusuf GT, Sellars ME, Deganello A et al. Retrospective Analysis of the Safety and Cost Implications of Pediatric Contrast-Enhanced Ultrasound at a Single Center. Am J Roentgenol 2016; 208: 446-452

[47] Sidhu PS, Cantisani V, Deganello A et al. Role of contrast-enhanced ultrasound (CEUS) in paediatric practice: An EFSUMB position statement. Ultraschall in Med 2017; 38: 33-43

[48] Education and Practical Standards Committee E. Minimal Training recommendations for the practice of medical ultrasound. Ultraschall in Med 2006; 27: 79-95

[49] Education and Practical Standards Committee E. Minimum Training Requirements for the Practice of Medical Ultrasound in Europe. Appendix 14: (CEUS) Contrast Enhanced Ultrasound. Ultraschall in Med 2010; 31: 426-427
[50] Jacobsen N, Nolsoe CP, Konge L et al. Contrast-Enhanced Ultrasound: Development of Syllabus for Core Theoretical and Practical Competencies. Ultrasound in Medicine \& Biology 2020; 46: 2287-2292

[51] Tay SY, Tiu CM, Hu B et al. Characterization and management of various renal cystic lesions by sonographic features. Journal of the Chinese Medical Association 2018; 81: 1017-1026

[52] Deganello A, Sellars ME, Yusuf GT et al. How Much Should I Record During a CEUS Examination? Practical Aspects of the Real-Time Feature of a Contrast Ultrasound Study. Ultraschall in Med 2018; 39: 484-486

[53] Correas JM, Claudon M, Tranquart F et al. The kidney: imaging with microbubble contrast agents. Ultrasound Q 2006; 22: 53-66

[54] Tsuruoka K, Yasuda T, Koitabashi K et al. Evaluation of Renal Microcirculation by Contrast-Enhanced Ultrasound With Sonazoid ${ }^{\mathrm{TM}}$ as a Contrast Agent Comparison Between Normal Subjects and Patients With Chronic Kidney Disease. Int Heart J 2010; 51: 176-182

[55] Narayanasamy S, Krishna S, Prasad Shanbhogue AK et al. Contemporary update on imaging of cystic renal masses with histopathological correlation and emphasis on patient management. Clinical Radiology 2019; 74: 83-94

[56] Park BK, Kim B, Kim SH et al. Assessment of cystic renal masses based on Bosniak classification: comparison of CT and contrast-enhanced US. Eur J Radiol 2007; 61: 310-314

[57] Chen Y, Wu N, Xue T et al. Comparison of contrast-enhanced sonography with MRI in the diagnosis of complex cystic renal masses. Journal of Clinical Ultrasound 2015; 43: 203-209

[58] Edenberg J, Gloersen K, Osman HA et al. The role of contrast-enhanced ultrasound in the classification of CT-indeterminate renal lesions. Scandinavian Journal of Urology 2016; 50: 445-451

[59] Bertolotto M, Cicero C, Perrone R et al. Renal masses with equivocal enhamcement at CT: characterization with contrast-enhanced ultrasound. Am J Roentgenol 2015; 205: W557-W565

[60] Siddaiah M, Krishna S, McInnes MDF et al. Is Ultrasound Useful for Further Evaluation of Homogeneously Hyperattenuating Renal Lesions Detected on CT? Am J Roentgenol 2017; 209: 604-610

[61] Gulati M, King KG, Gill IS et al. Contrast-enhanced ultrasound (CEUS) of cystic and solid renal lesions: a review. Abdominal Imaging 2015; 40: 1982-1996

[62] Defortescu G, Cornu JN, Bejar S et al. Diagnostic performance of contrast-enhanced ultrasonography and magnetic resonance imaging for the assessment of complex renal cysts: A prospective study. Int J Urol 2017; 24: 184-189

[63] Nicolau C, Bunesch L, Sebastia C. Renal complex cysts in adults: contrastenhanced ultrasound. Abdominal Imaging 2011; 36: 742-752

[64] Clevert DA, Minaifar N, Weckbach S et al. Multislice computed tomography versus contrast-enhanced ultrasound in evaluation of complex cystic renal masses using the Bosniak classification system. Clin Hemorheol Microcirc 2008; 39: 171-178

[65] Ragel M, Nedumaran A, Makowska-Webb J. Prospective comparison of use of contrast-enhanced ultrasound and contrast-enhanced computed tomography in the Bosniak classification of complex renal cysts. Ultrasound 2016; 24: 6-16

[66] Helenon O, Crosnier A, Verkarre $V$ et al. Simple and complex renal cysts in adults: Classification system for renal cystic masses. Diagnostic and Interventional Imaging 2018; 99: 189-218

[67] Ignee A, Straub B, Brix D et al. The value of contrast enhanced ultrasound (CEUS) in the characterisation of patients with renal masses. Clin Hemorheol Microcirc 2010; 46: 275-290

[68] Oon SF, Foley RW, Quinn D et al. Contrast-enhanced ultrasound of the kidney: a single-institution experience. Irish Journal of Medical Science (1971) 2018; 187: 795-802

[69] Israel GM, Bosniak MA. Pitfalls in Renal Mass Evaluation and How to Avoid Them. Radiographics 2008; 28: 1325-1338 
[70] Nicolau C, Bunesch L, Pano B et al. Prospective evaluation of CT indeterminate renal masses using US and contrast-enhanced ultrasound. Abdominal Imaging 2015; 40: 542-551

[71] Rubenthaler ], Mueller-Peltzer K, Negrúo de Figueiredo G et al. CEUS diagnostic workup of cystic renal lesions. Der Radiologe 2018; 58: 545552

[72] Xue LY, Lu Q, Huang B] et al. Contrast-enhanced ultrasonography for evaluation of cystic renal mass: in comparison to contrast-enhanced CT and conventional ultrasound. Abdominal Imaging 2014; 39: 1274-1283

[73] Rubenthaler J, Negrao de Figueiredo G, Mueller-Peltzer K et al. Evaluation of renal lesions using contrast-enhanced ultrasound (CEUS); a 10-year retrospective European single-centre analysis. European Radiology 2018; 28: 4542-4549

[74] Xu Y, Zhang S, Wei X et al. Contrast enhanced ultrasonography prediction of cystic renal mass in comparison to histopathology; 2014; 58: 429-438

[75] Destefani MH, Elias ] Jr, Serra Negra Trazzi AM et al. Minimally Complex Renal Cysts: Outcomes and Ultrasound Evaluation Compared with Contrast-Enhanced Cross-Sectional Imaging Bosniak Classification. Ultrasound in Medicine and Biology 2017; 43: 2167-2173

[76] Han HH, Choi KH, Oh YT et al. Differential diagnosis of complex renal cysts based on lesion size along with the Bosniak renal cyst classification. Yonsei Med J 2012; 53: 729-733

[77] Goenka AH, Remer EM, Smith AD et al. Development of a Clinical Prediction Model for Assessment of Malignancy Risk in Bosniak III Renal Lesions. Urology 2013; 82: 630-635

[78] Hindman NM. Approach to Very Small (< $1.5 \mathrm{~cm})$ Cystic Renal Lesions: Ignore, Observe, or Treat? Am J Roentgenol 2015; 204: 1182-1189

[79] Oh TH, Lee YH, Seo IY. Diagnostic Efficacy of Contrast-Enhanced Ultrasound for Small Renal Masses. Korean J Urol 2014; 55: 587-592

[80] Lam C], Kapoor A. The true malignancy risk of Bosniak III cystic renal lesions: Active surveillance or surgical resection? Can Urol Assoc J 2018; 2018/02/23: E276-E280

[81] Lang EK, Macchia RJ, Gayle B et al. CT-guided biopsy of indeterminate renal cystic masses (Bosniak 3 and 2F): accuracy and impact on clinical management. European Radiology 2002; 12: 2518-2524
[82] Sanz E, Hevia V, Gomez V et al. Renal Complex Cystic Masses: Usefulness of Contrast-Enhanced Ultrasound (CEUS) in Their Assessment and Its Agreement with Computed Tomography. Current Urology Reports 2016; 17: 89

[83] Clevert DA, Bensler S, Stickel M et al. Contrast enhanced ultrasound eases interpretation of an unclear renal tumor in addition to CT, MRI and histological findings - A case report in a young patient. Clin Hemorr Microcircul 2007; 36: 313-318

[84] Herts BR, Silverman SG, Hindman NM et al. Management of the Incidental Renal Masses;on CT: A White Paper of the ACR; Incidental Findings Committee. Journal of the American College of Radiology 2018; 15 : 264-273

[85] Hwang JH, Lee CK, Yu HS et al. Clinical Outcomes of Bosniak Category IIF Complex Renal Cysts in Korean Patients. Korean J Urol 2012; 2012/06/ 19: 386-390

[86] El-Mokadem I, Budak M, Pillai S et al. Progression, interobserver agreement, and malignancy rate in complex renal cysts (> Bosniak category IIF). Urologic Oncology: Seminars and Original Investigations 2014; 32: 24

[87] O’Malley RL, Godoy G, Hecht EM et al. Bosniak Category IIF Designation and Surgery for Complex Renal Cysts. Journal of Urology 2009; 182: 1091-1095

[88] Smith AD, Remer EM, Cox KL et al. Bosniak Category IIF and III Cystic Renal Lesions: Outcomes and Associations. Radiology 2012; 262: 152 160

[89] Graumann O, Osther SS, Karstoft ] et al. Evaluation of Bosniak category IIF complex renal cysts. Insights Imaging 2013; 4: 471-480

[90] Adey GS, Pedrosa I, Rofsky NM et al. Lower Limits of Detection Using Magnetic Resonance Imaging for Solid Components in Cystic Renal Neoplasms. Urology 2008; 71: 47-51

[91] Lan D, Qu HC, Li N et al. The Value of Contrast-Enhanced Ultrasonography and Contrast-Enhanced CT in the Diagnosis of Malignant Renal Cystic Lesions: A Meta-Analysis. PLoS ONE 2016; 11: e0155857 